\title{
Одномодовые лазеры (1050 нм) мезаполосковой конструкции на основе гетероструктуры AlGaAs/GaAs со сверхузким волноводом
}

\author{
(C) И.С Шашкин, А.Ю Лешко, Д.Н Николаев, В.В Шамахов, Д.А Веселов, Н.А Рудова, К.В Бахвалов, \\ В.В Золотарев, С.О Слипченко, Н.А Пихтин, П.С Копьев
}

Физико-технический институт им. А.Ф. Иофрфе Российской академии наук, 194021 Санкт-Петербург, Россия

E-mail: shashkin@mail.ioffe.ru

Поступила в Редакцию 12 декабря 2019 г.

В окончательной редакции 23 декабря 2019 г.

Принята к публикации 23 декабря 2019 г.

\begin{abstract}
Исследованы излучательные характеристики одномодовых лазеров на основе гетероструктуры $\mathrm{AlGaAs} / \mathrm{GaAs}$ со сверхузким волноводом. Показано, что использование сверхузкого волновода (100 нм) и тонких $(70 \mathrm{Hм})$ широкозонных барьеров на границе волновод/эмиттер позволяет решать задачу сужения расходимости излучения в дальней зоне в плоскости, перпендикулярной слоям гетероструктуры. В разработанных одномодовых лазерах с узким мезаполосковым контактом (ширина 5.1 мкм) расходимость излучения в плоскости, параллельной и перпендикулярной слоям гетероструктуры, составила 5 и $18.5^{\circ}$ соответственно. Разработанные лазеры демонстрируют эффективную работу в одномодовом режиме до мощности 200 мВт, а дальнейшее повышение тока приводит к включению дополнительных мод высшего порядка, при этом достигаемая максимальная мощность в непрерывном режиме ограничена перегревом и составляет 550 мВт. Переход в импульсный режим генерации (длительность импульсов 200 нс) позволил поднять максимальную пиковую мощность до 1500 мВт.
\end{abstract}

Ключевые слова: одномодовый лазер, сверхузкий волновод, $\mathrm{AlGaAs} / \mathrm{GaAs}$, моды высшего порядка, пиковая мощность.

DOI: $10.21883 /$ FTP.2020.04.49150.9334

\section{1. Введение}

В последние годы наблюдается заметный прогресс в области лазерных технологий, связанный с повышением мощности и эффективности источников лазерного излучения на основе волоконных и твердотельных лазеров. Одной из причин является заметное улучшение излучательных характеристик полупроводниковых лазеров, используемых как источники оптической накачки активных сред. Так, продемонстрированы непрерывные оптические мощности на уровне 30 Вт для одиночных многомодовых полупроводниковых лазеров [1]. Однако существенным недостатком таких источников является плохое качество лазерного луча и, как следствие, низкая яркость источников, работающих в многомодовом режиме. Одно из решений связано с разработкой мощных одномодовых полупроводниковых лазеров, которые в дальнейшем можно объединять в линейки и решетки [2]. При решении поставленной задачи одним из факторов, определяющих простоту и эффективность оптической схемы сбора излучения источников накачки, является минимальное значение астигматизма, выражающегося в различии расходимости излучения для плоскости параллельной и перпендикулярной слоям гетероструктуры. Для решения данной задачи использовался ряд различных подходов к конструированию поперечного волновода, формируемого конструкцией гетероструктуры. К таким подходам можно отнести конструкцию гетероструктуры типа SCWOL (slab-coupled-optical-waveguide- laser) с толщиной поперечного волновода 3.9 мкм, где для лазеров с узким полосковым контактом (ширина 5 мкм) продемонстрированы расходимость в плоскости, перпендикулярной слоям гетероструктуры $11.4^{\circ}$ (здесь и далее речь будет идти о расходимости на уровне половины от максимума $F W H M)$, и оптическая мощность 1 Вт [3]. Использование сверхширокого волновода толщиной 8.6 мкм позволило снизить расходимость до $9^{\circ}$, при этом увеличение ширины полоска до 7 мкм позволило достичь 1.3 Вт выходной оптической мощности [4]. Здесь важно отметить, что в формирование моды в таком широком волноводе вносила вклад активная область, сформированная четырьмя InGaAs квантовыми ямами и компенсирующими GaAsP барьерами. Отметим работу по оптимизации сверхширокого волновода, в частности в [5] удалось получить расходимость $11.5^{\circ}$ при выходной оптической мощности 1.9 Вт, при ширине полоска 7 мкм. Отдельно можно выделить подход, основанный на использовании одномерных фотонных кристаллов [6]: толщина структуры 16 мкм, $F W H M=14^{\circ}$, $P=1.9 \mathrm{Bт,}, w=9$ мкм. Видно, что общей чертой всех предложенных решений является использование сверхшироких волноведущих слоев, так как расходимость излучения связана с областью локализации лазерной моды. Вместе с этим добиться широкой области локализации моды можно в сверхузких волноводах, для которых толщина существенно меньше длины волны в веществе. Использование сверхузких волноводов, с одной стороны, должно позволить снизить общую толщину 
структуры, а с другой - избавить от необходимости решения проблемы селекции мод высших порядков, так как сверхширокие волноводы в основном являются моногомодовыми. В рамках данной работы представлены результаты исследований одномодовых лазеров с узким мезаполосковым контактом на основе гетероструктры со сверхузким волноводом.

\section{2. Моделирование}

Для выбора дизайна гетероструктуры на первом этапе было проведено моделирование, демонстрирующее связь расходимости излучения в плоскости, перпендикулярной слоям гетероструктуры, с параметрами волноводного слоя. Для определенности оптимизация проводилась в рамках эмиттеров на основе $\mathrm{Al}_{x} \mathrm{Ga}_{1-x} \mathrm{As}(x=0.3)$, волновода на основе GaAs и InGaAs квантовой ямы толщиной 9 нм, симметрично расположенной в волноводе. На рис. 1 показана зависимость расходимости излучения на уровне половина от максимума от толщины волновода и зависимость фактора оптического ограничения Г в активной области. Видно, что максимумы зависимостей не совпадают, так максимальная расходимость наблюдается для волновода толщиной 0.5 мкм, дальнейшее снижение толщины волновода приводит к снижению расходимости, а максимальный фактор оптического ограничения в активной области достигается при толщине волновода 0.25 мкм, что позволяет сохранить приемлемое значение усиления на пороге генерации даже в случае тонких волноводов. Здесь стоит заметить, что предельным случаем сверхузкого волновода являются гетероструктуры с волноводами на основе квантовой ямы [7]. В таких гетероструктурах при электрической накачке была продемонстрирована расходимость $15^{\circ}$ для одиночной квантовой ямы [7] и $10^{\circ}$ для волновода на основе 6 квантовых ям [8], однако отсутствие барьеров приводило к существенной утечке носителей, что выражалось в низком значении внутреннего квантового выхода, составившего 70\%. Это значит, что переход к сверхузким волноводам может сопровождаться утечками носителей заряда. Для решения данной проблемы конструкция гетероструктуры была дополнена тонкими широкозонными барьерами на основе $\mathrm{Al}_{x} \mathrm{Ga}_{1-x} \mathrm{As}(x=0.4)$, расположенными на границе волновод-эмиттер. Для исследования влияния толщины барьера на волноводные характеристики были проведены расчеты факторов оптического ограничения и расходимости для барьеров толщиной 30 и 70 нм (рис. 1). Видно, что использование тонких барьеров приводит к усилению эффекта делокализации моды при одинаковых толщинах волноводных слоев, что выражается в уменьшении расходимости излучения в плоскости, перпендикулярной слоям гетероструктуры, в структурах с тонкими широкозонными барьерами по сравнению со структурами, в которых эти барьеры отсутствуют. Кроме этого увеличение толщины барьера с 30 до 70 нм усиливает эффект делокализации моды.

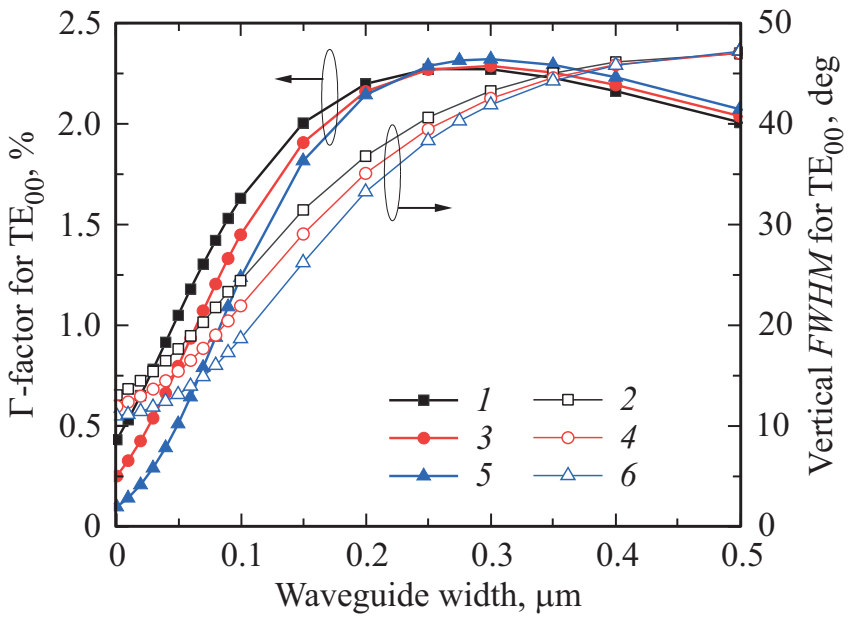

Рис. 1. Расчетная зависимость фактора оптического ограничения и расходимости излучения на уровне половина от максимума от толщины волновода для структур без широкозонных барьеров $(1,2)$ и структур с широкозонными барьерами 30 нм $(3,4)$ и 70 нм $(5,6)$.

Как отмечалось выше, если рассматривать $15^{\circ}$ как предельное значение расходимости, к которому стремится структура с волноводом, сформированным только одной квантовой ямой, то снижение толщины волновода до нескольких десятков нм не должно привести к существенному падению расходимости. Таким образом, исходя из проведенного анализа были выбраны толщины волновода и барьеров 100 и 70 нм соответственно, что обеспечивает расходимость $18.5^{\circ}$ и сохраняет высокое значение фактора оптического ограничения в активной области.

\section{3. Экспериментальные исследования}

Для экспериментальной реализации был выбран следующий дизайн гетероструктуры: $n$ - и $p$-эмиттеры $\mathrm{Al}_{x} \mathrm{Ga}_{1-x} \mathrm{As}(x=0.3)$ толщиной 2.75 мкм каждый, $n$ и $p$ широкозонные барьеры $\mathrm{Al}_{x} \mathrm{Ga}_{1-x} \mathrm{As}(x=0.4)$ толщиной 70 нм каждый, нелегированный GaAs-волновод толщиной 100 нм, активная область InGaAs толщиной 9 нм. Схематическое изображение гетероструктуры показано на рис. 2. Структура была выращена методом МОСгидридной эпитаксии. Для характеризации оптических свойств из гетероструктуры были изготовлены полупроводниковые лазеры с широким (100 мкм) и узким (5.1 мкм) мезаполосковым контактом. Для лазеров с широким мезаполосковым контактом была использована конструкция глубокая меза, в которой мезаканавки пересекали волноводный слой, что позволило избежать эффекта растекания тока, что важно при определении пороговой плотности тока. Для лазеров с узким мезаполосковым контактом глубина травления была выбрана на основании расчета модовой структуры для 2D-волновода, учитывающего усиление в области инжекции и 
поглощение в пассивной области. На рис. 3 приведены расчетные значения модального усиления для мод $\mathrm{TE}_{00}$, $\mathrm{TE}_{10}, \mathrm{TE}_{20}$ от величины остаточной толщины $p$-эмиттера (RLT - Residual layer thickness; RLT $=W-D$, где $W$ - глубина мезаканавки, $D$ - суммарная толщина p-эмиттера и контактного слоя). Видно, что выбор оптимальной глубины травления мезаканавки обеспечивает дискриминацию мод высшего порядка относительно нулевой моды за счет более высокого значения модального усиления. Для оптимальной глубины травления модальное усиление моды $\mathrm{TE}_{00}$ выше на величину $\sim 10 \%$ по сравнению с остальными модами. Таким образом, была выбрана следующая расчетная конструкция одномодо-

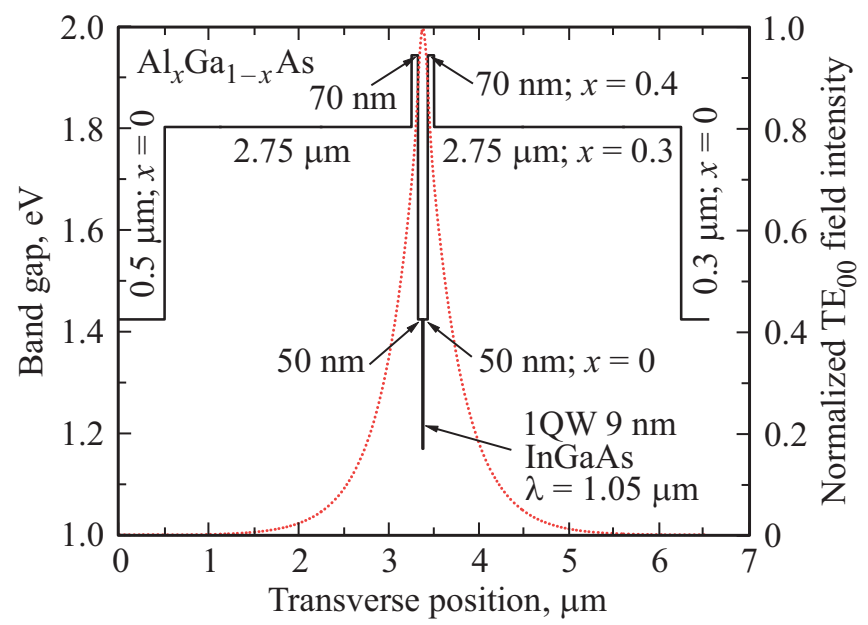

Pис. 2. Схематическое изображение гетероструктуры с тонкими широкозонными барьерами и поперечный профиль интенсивности нулевой моды.

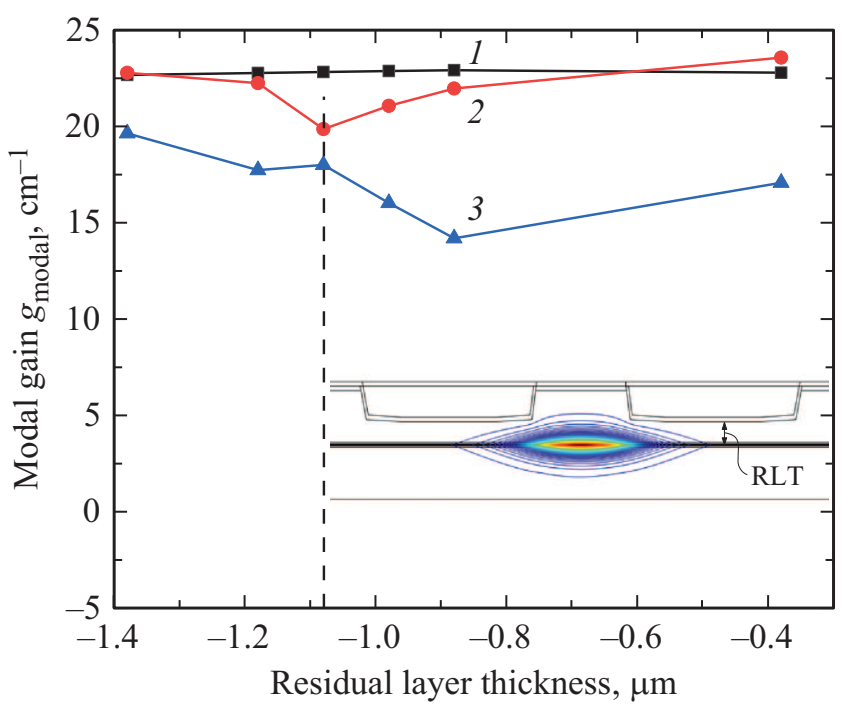

Рис. 3. Расчетная зависимость модального усиления мод $\mathrm{TE}_{00}, \mathrm{TE}_{10}, \mathrm{TE}_{20}$ для 2D-волновода при различных значениях параметра „residual layer thickness“ (RLT). На вставке профиль $\mathrm{TE}_{00}$ моды 2D-волновода.

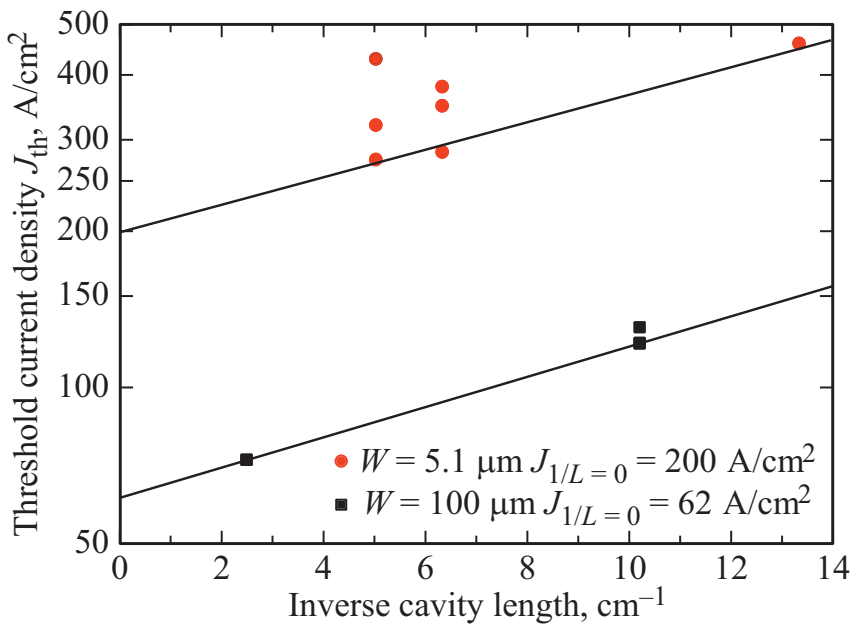

Рис. 4. Зависимости пороговой плотности тока от обратной длины резонатора. Квадраты - экспериментальные значения для лазеров конструкции глубокая меза с шириной 100 мкм; круги - экспериментальные значения для лазеров конструкции мелкая меза с шириной 5.1 мкм.

вых лазеров: ширина мезаполоскового контакта 5.1 мкм, $\mathrm{RLT}=-1.08$ мкм.

Все измерения проведены при температуре теплоотвода $25^{\circ} \mathrm{C}$. На рис. 4 показаны зависимости пороговой плотности тока от обратной длины резонатора. Видно, что пороговый ток при бесконечной длине резонатора достигает $62 \mathrm{~A} / \mathrm{cm}^{2}$ для лазеров с широким контактом, конструкции глубокая меза, в которой растекание отсутствует. В лазерах с узким мезаполосковым контактом пороговый ток при бесконечной длине резонатора достигает $200 \mathrm{~A} / \mathrm{cm}^{2}$, что свидетельствует о вкладе латерального растекания тока за счет недотрава до волновода. Из зависимостей обратной дифференциальной эффективности от длины резонатора для лазеров с широким и узким мезаполосковым контактом были определены внутренние оптические потери и внутренний квантовый выход. Было установлено, что внутренние оптические потери для лазеров обеих конструкций достигают $1.3 \mathrm{~cm}^{-1}$. Внутренний квантовый выход для лазеров с широким мезаполосковым контактом составил 97\%, что демонстрирует эффективность использования тонких широкозонных барьеров на границе волновод-эмиттер для подавления утечки носителей заряда. При этом внутренний квантовый выход для одномодовых лазеров составил 87\%, что, по нашему мнению, может быть связано с вкладом растекания тока.

На рис. 5 показана ватт-амперная характеристика в непрерывном режиме генерации, полученная для полупроводниковых лазеров с естественносколотыми гранями и нанесенными на грани резонатора просветляющими и отражающими покрытиями. Из полученных зависимостей видно, что „кинк“ происходит в диапазоне токов 300-350 мА, независимо от коэффициента отражения зеркал резонатора. При уменьшении длины резонатора 

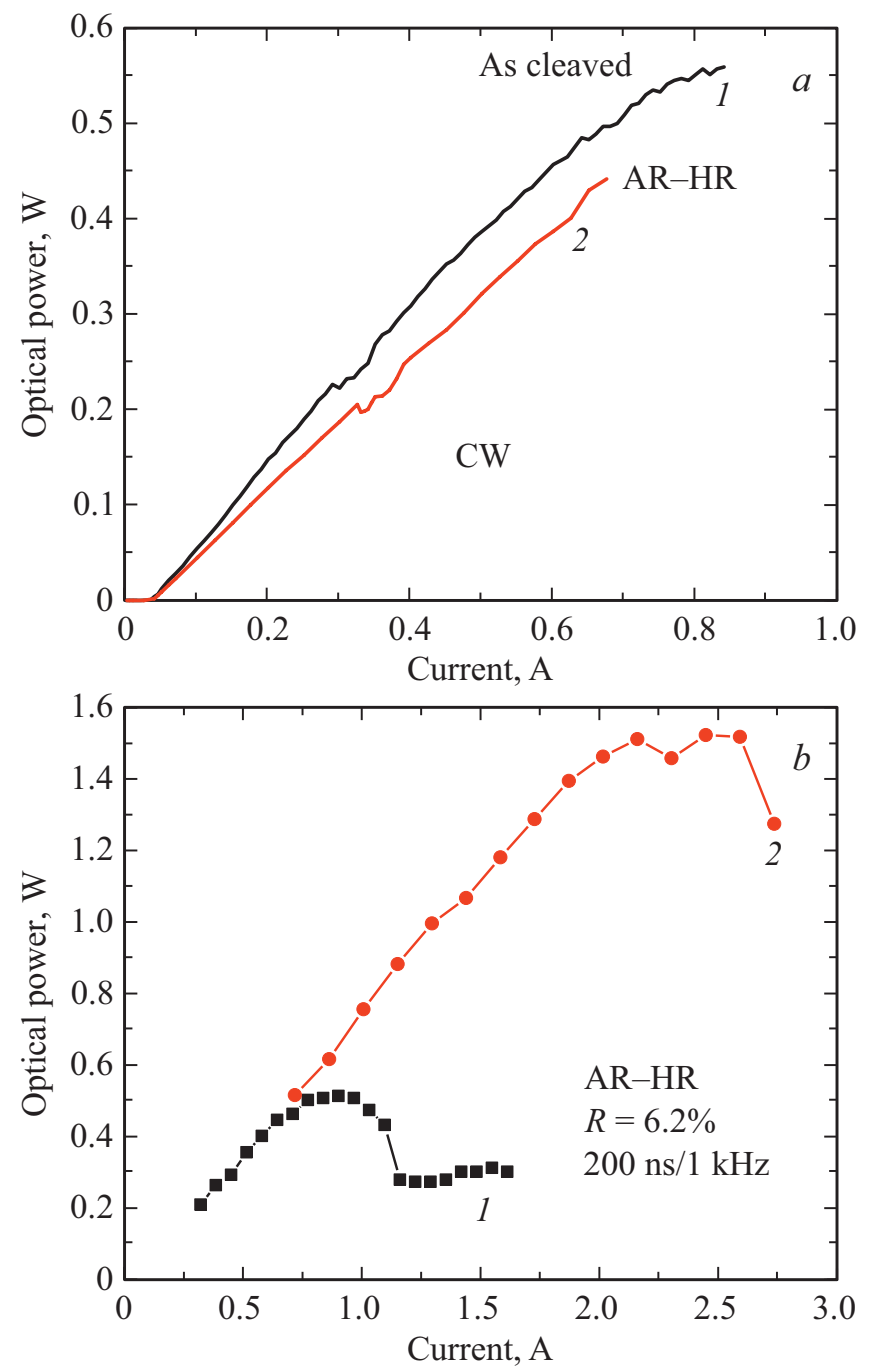

Рис. 5. $a-$ ватт-амперные характеристики в непрерывном режиме генерации для полупроводниковых лазеров конструкции мелкая меза с естетсвенносколотыми гранями (1) и нанесенными на грани резонатора просветляющими (6.2\%) и отражающими (95\%) покрытиями (2). $b-$ ватт-амперные характеристики в импульсном режиме генерации для полупроводниковых лазеров конструкции мелкая меза с нанесенными на грани резонатора просветляющими $(6.2 \%)$ и отражающими (95\%) покрытиями для момента времени 20 нс (1) и 175 нс (2) от начала импульса. Длина резонатора 2 мм.

область возникновения „кинка“ смещалась в диапазон меньших токов накачки, например для длины резонатора 780 мкм „кинк“ проявлялся на токах 150-200 мА. Из приведенных на рис. 5, a зависимостей видно, что максимальная оптическая мощность была ограничена насыщением ВТАХ и находилась на уровне 0.5 Вт. По нашему мнению, насыщение ВTAX в непрерывном режиме связано с тепловыми эффектами, что приводит к разогреву активной области и ухудшению излучательных характеристик.

Другой важной характеристикой одномодовых лазеров является дальнее поле. На рис. 6 показано распределе- ние излучения в дальней зоне, в плоскости, параллельной и перпендикулярной $p-n$-переходу. Измерения показали, что для перпендикулярной плоскости (рис. 6,a) форма поля не меняется с током накачки и составляет $18.5^{\circ}$ на уровне половина от максимума. Полученное значение совпадает с расчетным (рис. 1 и 6,a) Для параллельной плоскости распределение поля сохраняет гауссову форму с характерными низкоинтенсивными „ушами“ для токов до „кинка“ на ВтАХ (рис. 6, $b$ ). Это может свидетельствовать о вкладе мод высшего порядка. В работе [9] было показано, что незначительное присутствие мод высшего порядка не существенно сказывается на качестве модовой структуры лазерного излучения и позволяет сохранить величину $M^{2}$ в диапазоне $1-1.4$. Однако форма дальнего параллельного поля заметно меняется, когда ток накачки становится выше „кинка“ ВтАХ (рис. 6, $b$ ). Видно, что распределение смещается
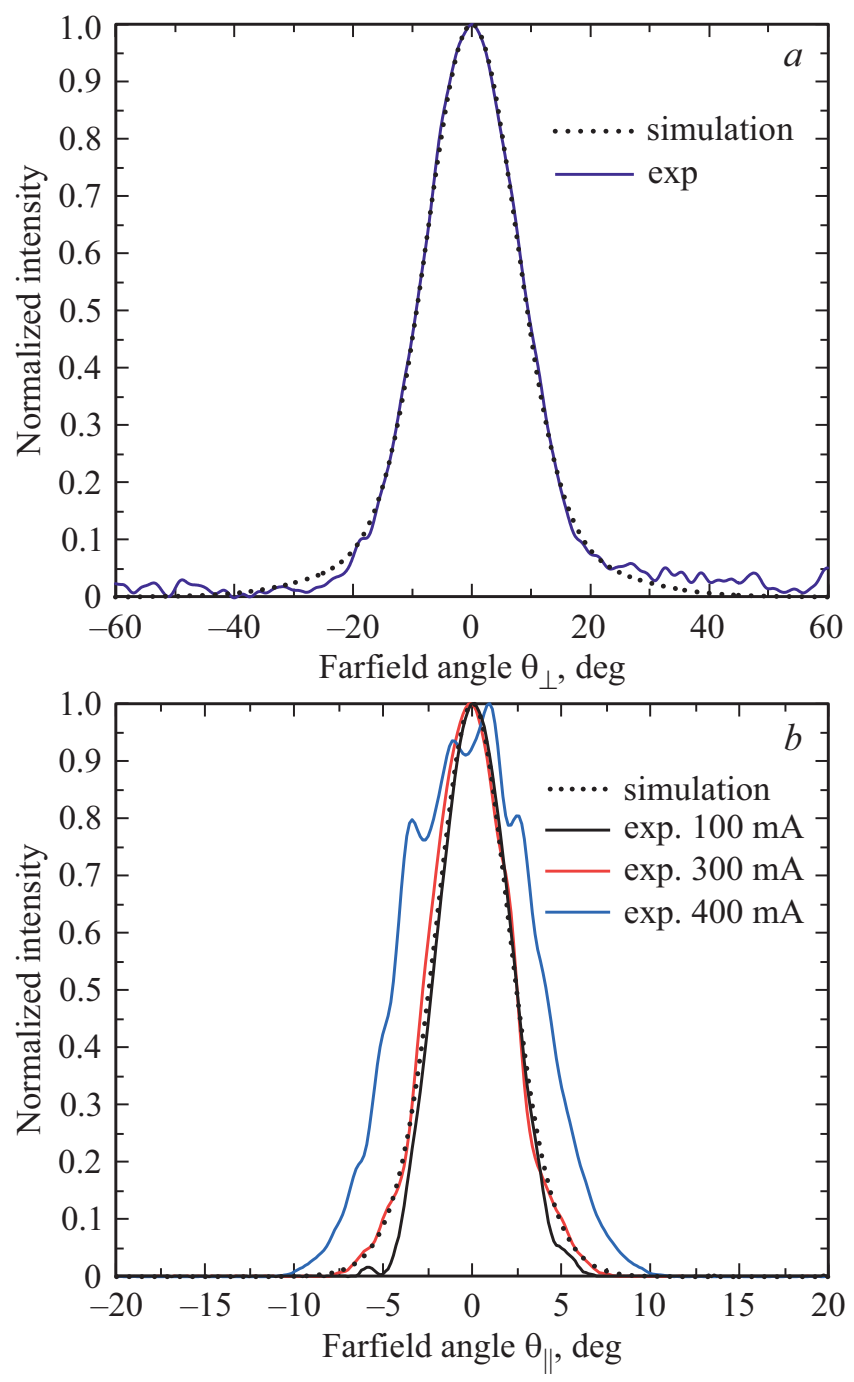

Рис. 6. Распределение излучения в дальней зоне для полупроводниковых лазеров конструкции мелкая меза, работающих в непрерывном режиме генерации, в плоскости перпендикулярной $(a)$ и параллельной $(b) p-n$-переходу. Длина резонатоpa 2 мм. 


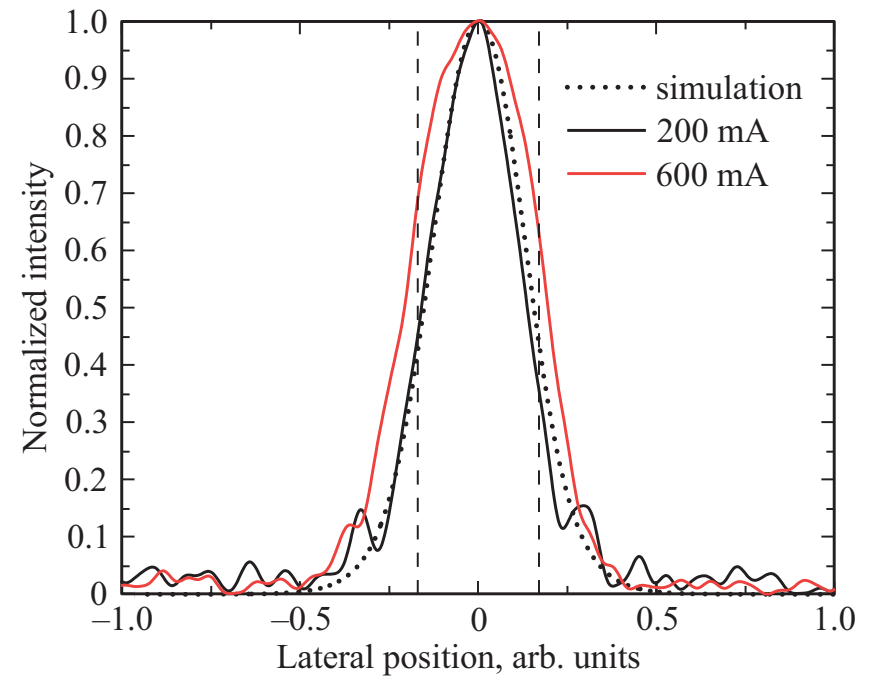

Рис. 7. Распределения излучения в ближней зоне, полученные из расчета и экспериментально измеренные, в плоскости, параллельной $p-n$-переходу, для полупроводниковых лазеров конструкции мелкая меза, работающих в непрерывном режиме генерации. Длина резонатора 2 мм. Пунктирные линии показывают границы мезаполоска из расчета.

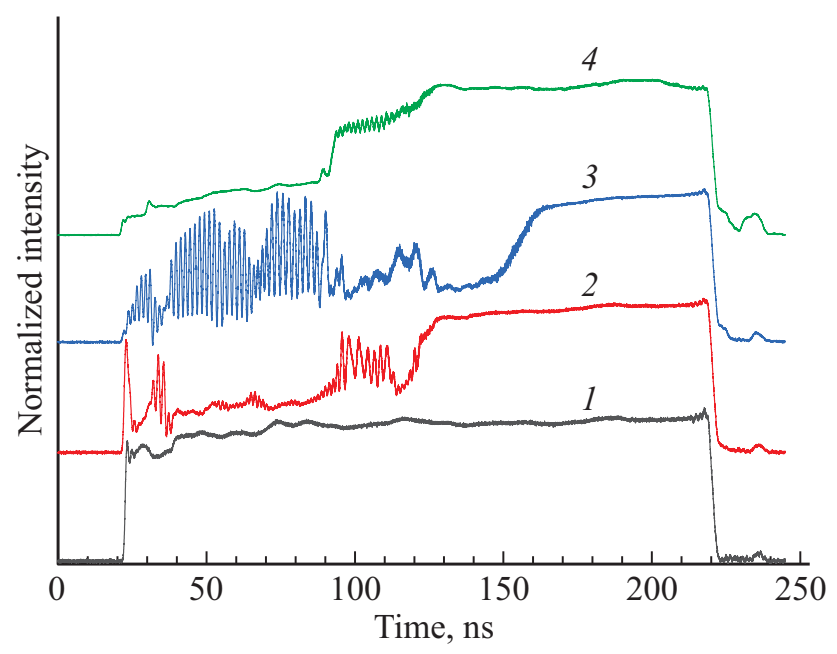

Рис. 8. Зависимость оптической мощности от времени для полупроводникового лазера с длиной резонатора 2 мм и нанесенными просветляющими и отражающими покрытиями. Среднее значение амплитуды тока между моментами времени 20 и 175 нс от начала импульса, мА: $1-600,2-800,3-1200$, $4-1600$.

относительно центрального положения и возникает модуляция максимума (рис. 6, $b$ ). Измерения распределения ближнего поля показали, что форма ближнего поля сохраняется при токах накачки до „кинка“, дальнейшее повышение тока приводит к уширению, что демонстрирует вклад мод высшего порядка (рис. 7).

Для оценки максимальной мощности были проведены измерения в импульсном режиме генерации. Переход к импульсному режиму обеспечивает снижение тепловой нагрузки на лазерный кристалл и, таким образом позволяет провести измерения до больших значений тока накачки. В проведенном эксперименте использовался источник накачки с длительностью импульса 200 нс. На рис. 8 показаны треки, отражающие динамику выходной оптической мощности полупроводникового лазера с длиной резонатора 2 мм и нанесенными просветляющими и отражающими покрытиями. Установлено, что в диапазоне токов до 600 мА форма оптического импульса с достаточной степенью точности повторяет форму импульса тока. Однако дальнейшее увеличение тока приводило к заметному искажению формы лазерного импульса (рис. 8 , токи более 800 мА). Видно, что началу импульса соответствует низкоэффективный режим генерации и только с течением времени эффективность лазерной генерации восстанавливается. При этом восстановление происходит достаточно резко, что свидетельствует о пороговом характере процесса. Другая особенность динамики лазерной генерации выражалась в движении границы перехода от низкоэффективного к высокоэффективному режимам генерации. Из приведенных форм импульсов (рис. 8) видно, что с ростом тока накачки фронт перехода между режимами смещается к концу импульса. Дальнейшее увеличение тока сопровождается движением фронта к началу импульса. Однако полностью избежать области низкоэффективной работы не удается и в области максимальных токов движение фронта прекращается. На рис. 5, $b$ показаны зависимости пиковой оптической мощности от амплитуды тока накачки, взятые для моментов времени 20 нс (что соответствует области низкоэффективной генерации) и $175 \mathrm{Hc}$ от начала импульса. Видно, что в области высокоэффективной генерации максимальная мощность достигает 1500 мВт при амплитуде тока 2100 мА. Известен ряд работ, посвященных исследованиям нетепловых эффектов, определяющих падение излучательной эффективности многомодовых [10,11] и одномодовых [12] полупроводниковых лазеров. Было показано, что основными факторами являются включение мод с низкими потерями на выход в многомодовых лазерах [10,11], так называемых замкнутых модовых структур, а также коллапс латерального волновода в одномодовых лазерах [12]. Кроме этого было зафиксировано, что в области низкоэффективной лазерной генерации существуют режимы, характеризующиеся достаточно сложными и быстрыми периодически повторяющимися переходными процессами. Подробные исследования особенностей работы лазера в данном режиме будут проводиться в следующих работах.

\section{4. Заключение}

В работе показано, что использование сверхузких волноводов с широкозонными барьерами позволяет решать задачу сужения расходимости излучения в дальней зоне в плоскости, перпендикулярной слоям гетероструктуры, при этом использование тонких широкозонных барье- 
ров способствует снижению расходимости. Разработанные лазеры с узким мезаполосковым контактом демонстрируют эффективную работу в одномодовом режиме до мощности $200 \mathrm{MB}$, а дальнейшее повышение тока приводит к включению дополнительных мод высшего порядка, при этом достигаемая максимальная мощность в непрерывном режиме ограничена перегревом и составляет 550мВт для образцов без интерференционных покрытий. Переход в импульсный режим генерации позволил поднять пиковую мощность до 1500 мВт.

\section{Финансирование работы}

Исследование выполнено за счет гранта Российского научного фонда (проект № 19-79-30072)

\section{Конфликт интересов}

Авторы заявляют, что у них нет конфликта интересов.

\section{Список литературы}

[1] V. Gapontsev, N. Moshegov, I. Berezin, A. Komissarov, P. Trubenko, D. Miftakhutdinov, I. Berishev, V. Chuyanov, O. Raisky, A. Ovtchinnikov. In: Proc. SPIE, High-Power Diode Laser Technology XV, ed. by M.S. Zediker (USA, 2017) v. 10086, p. 1008604.

[2] R. Platz, B. Eppich, J. Rieprich, W. Pittroff, G. Erbert, P. Crump. High Power Laser Sci. Eng., 4, e3 (2016).

[3] R.K. Huang, J.P. Donnelly, L.J. Missaggia, C.T. Harris, J. Plant, D.E. Mull, W.D. Goodhue. IEEE Photon. Technol. Lett., $15(7), 900$ (2003).

[4] A. Pietrzak, H. Wenzel, P. Crump, F. Bugge, J. Fricke, M. Spreemann, G. Erbert, G. Tränkle. IEEE J. Quant. Electron., 48 (5), 568 (2012).

[5] S. Zhao, A. Qi, M. Wang, H. Qu, Y. Lin, F. Dong, W. Zheng. Opt. Express, 26 (3), 3518 (2018).

[6] M.J. Miah, T. Kettler, K. Posilovic, V.P. Kalosha, D. Skoczowsky, R. Rosales, D. Bimberg, J. Pohl, M. Weyers. Appl. Phys. Lett., 105 (15), 151105 (2014).

[7] С.О. Слипченко, А.А. Подоскин, Н.А. Пихтин, А.Ю. Лешко, А.В. Рожков, И.С. Тарасов. Письма ЖТФ, 39 (8), 9 (2013).

[8] Н.В. Дикарева, Б.Н. Звонков, И.В. Самарцев, С.М. Некоркин, Н.В. Байдусь, А.А. Дубинов. ФТП, 53 (12), 1718 (2019).

[9] M. Wilkens, H. Wenzel, J. Fricke, A. Maabdorf, P. Ressel, S. Strohmaier, A. Knigge, G. Erbert, G. Trankle. IEEE Photon. Technol. Lett., 30 (6), 545 (2018).

[10] С.О. Слипченко, А.А. Подоскин, Д.А. Винокуров, А.Л. Станкевич, А.Ю. Лешко, Н.А. Пихтин, В.В. Забродский, И.С. Тарасов. ФТП, 45 (10), 1431 (2011).

[11] S.O. Slipchenko, A.A. Podoskin, N.A. Pikhtin, I.S. Tarasov. Laser Phys., 24 (10), 105001 (2014).

[12] A. Komissarov, M. Maiorov, R. Menna, S. Todorov, J. Connol1y, D. Garbuzov, V. Khalfin, A. Tsekoun. In: Technical Digest. Summaries of papers presented at the Conference on Lasers and Electro-Optics. Postconference Technical Digest [IEEE Cat. No.01CH37170] (2001) p. 31.

Редактор А.Н. Смирнов

\section{Single-mode lasers $(1050 \mathrm{~nm})$ of a mesa-stripe design based on AIGaAs/GaAs heterostructure with ultra-narrow waveguide}

\author{
I.S. Shashkin, A.Y. Leshko, D.N. Nikolaev, \\ V.V. Shamakhov, D.A. Veselov, N.A. Rudova, \\ K.V. Bakhvalov, V.V. Zolotarev, S.O. Slipchenko, \\ N.A. Pikhtin, P.S. Kop'ev \\ loffe Institute, \\ 194021 St. Petersburg, Russia
}

Abstract The radiation properties of single-mode lasers based on an $\mathrm{AlGaAs} / \mathrm{GaAs}$ heterostructure with an ultra-narrow waveguide are studied. It is shown that the use of an ultranarrow waveguide $(100 \mathrm{~nm})$ and thin $(70 \mathrm{~nm})$ wide-gap barriers at the waveguide/cladding interface improves the vertical divergence. The lateral and vertical divergences were 5 and 18.5 degrees, respectively, for the developed narrow-stripe $(5.1 \mu \mathrm{m})$ single-mode lasers. An efficient single-mode operation up to a power of $200 \mathrm{~mW}$ is demonstrated. A further increase in current leads to the onset of higher-order modes, while the maximum optical power achieved under continuous-wave $(\mathrm{CW})$ operation is limited by overheating and is $550 \mathrm{~mW}$. The transfer to a pulsed operation (pulse width $200 \mathrm{~ns}$ ) made it possible to raise the maximum peak optical power to $1500 \mathrm{~mW}$. 